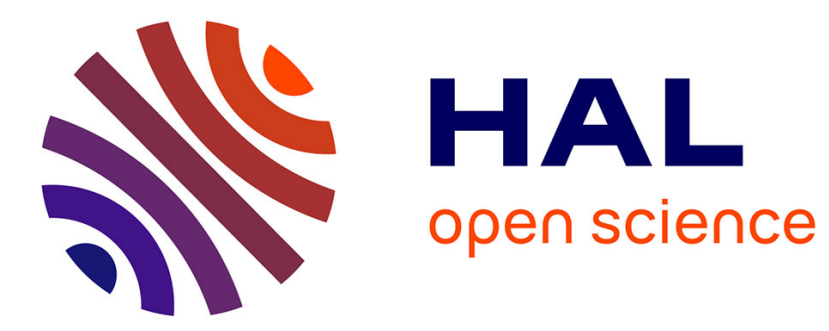

\title{
Comportement non newtonien des suspensions de particules browniennes
}

P. Mills, P. Snabre, D. Quemada

\section{To cite this version:}

P. Mills, P. Snabre, D. Quemada. Comportement non newtonien des suspensions de particules browniennes. Revue de Physique Appliquée, 1981, 16 (9), pp.523-527. 10.1051/rphysap:01981001609052300 . jpa-00244945

\section{HAL Id: jpa-00244945 https://hal.science/jpa-00244945}

Submitted on 1 Jan 1981

HAL is a multi-disciplinary open access archive for the deposit and dissemination of scientific research documents, whether they are published or not. The documents may come from teaching and research institutions in France or abroad, or from public or private research centers.
L'archive ouverte pluridisciplinaire HAL, est destinée au dépôt et à la diffusion de documents scientifiques de niveau recherche, publiés ou non, émanant des établissements d'enseignement et de recherche français ou étrangers, des laboratoires publics ou privés. 


\title{
Comportement non newtonien des suspensions de particules browniennes
}

\author{
P. Mills, P. Snabre et D. Quemada \\ Laboratoire de Biorhéologie et d'Hydrodynamique Physiologique (*), U.E.R. de Physique, Université Paris VII, \\ 2, place Jussieu 75251 Paris Cedex 05, France
}

(Reçu le 3 mars 1981, révisé le 10 juin 1981, accepté le 12 juin 1981)

\begin{abstract}
Résumé. - Les résultats expérimentaux concernant la viscosité apparente de suspensions de sphères dures, de rayon inférieur au micron, en fonction du taux de cisaillement, mettent en évidence le caractère non newtonien de telles suspensions.

La viscosité apparente décroît avec le taux de cisaillement, et cet effet est d'autant plus marqué que la suspension est concentrée. Dans cet article, nous supposons que le mécanisme responsable de l'augmentation de la viscosité apparente pour de faibles valeurs du taux de cisaillement est l'écart, dû au mouvement brownien, entre la microrotation des particules et la vorticité locale du fluide. L'expression théorique de la viscosité apparente, pour une concentration donnée, s'exprime alors en fonction de la viscosité de cisaillement, de la viscosité de rotation et d'une contrainte réduite. Elle coîncide remarquablement avec une expression empirique, antérieurement proposée, afin d'ajuster les résultats expérimentaux.
\end{abstract}

\begin{abstract}
Experimental results related to the apparent viscosity of hard sphere dispersions, with a sphere radius below one micron, versus shear rate reveal the non newtonian behaviour of such dispersions.

The system behaves as a shear thinning fluid; the sharper this effect, the more concentrated the dispersion. In this paper, the apparent viscosity increase in the low shear range is supposed to be connected to the difference, due to brownian motion, between the particle microrotation and the local fluid vorticity. Theoretical expression of the apparent viscosity is then expressed versus shear viscosity, rotation viscosity, and reduced shear stress. This expression coincides very well with an empirical expression used up to now to fit experimental data.
\end{abstract}

Les suspensions colloïdales et les solutions macromoléculaires en écoulement ont souvent un comportement non newtonien. Un concept souvent utilisé pour modéliser de tels fluides est le modèle de sphères dures. Les dimensions de ces sphères sont assez grandes, comparées aux dimensions des molécules du fluide suspendant, et néanmoins assez petites pour que les mouvements browniens de translation et de rotation soient assez importants relativement aux mouvements de ces particules dus à l'écoulement du fluide environnant. L'influence du mouvement brownien sera d'autant plus nette que d'autres processus irréversibles, tels que la sédimentation des particules, seront prévenus. Nous supposerons donc la densité des sphères dures ajustée à celle du milieu suspendant.

La technologie permettant la réalisation de suspensions de sphères dures monodispersées comme les latex, dispersions en phase aqueuse de polymères synthétiques émulsionnés et polymérisés, a été développée ces dernières années [1] et a permis des études rhéologiques précises de suspensions s'approchant

$\left(^{*}\right)$ Equipe de Recherche Associée du C.N.R.S. du modèle théorique. Si nous considérons les résultats expérimentaux concernant la viscosité apparente de suspensions de particules browniennes, nous constatons que cette viscosité varie considérablement avec le taux de cisaillement $\dot{\gamma}$. Nous avons choisi pour illustrer ceci un exemple tiré d'un article de Krieger et Dougherty [2] (Fig. 1).

Par contre, lorsque la taille des particules est assez importante pour que le mouvement brownien puisse être négligé, on constate que la viscosité d'une suspension de sphères dures est pratiquement indépendante du taux de cisaillement. Cette dernière constatation est tout à fait cohérente avec les observations de Mason concernant la réversibilité des collisions de sphères dures [3]. L'écoulement stationnaire de telles sphères étant réversible, il est normal que la viscosité d'une telle suspension soit indépendante du temps, donc de la valeur du taux de cisaillement. Inversement l'introduction d'un processus irréversible tel que le mouvement brownien pourra entrainer une dépendance entre la viscosité et le taux de cisaillement.

La remarque précédente permet de prédire que la valeur stationnaire de la viscosité obtenue pour des 


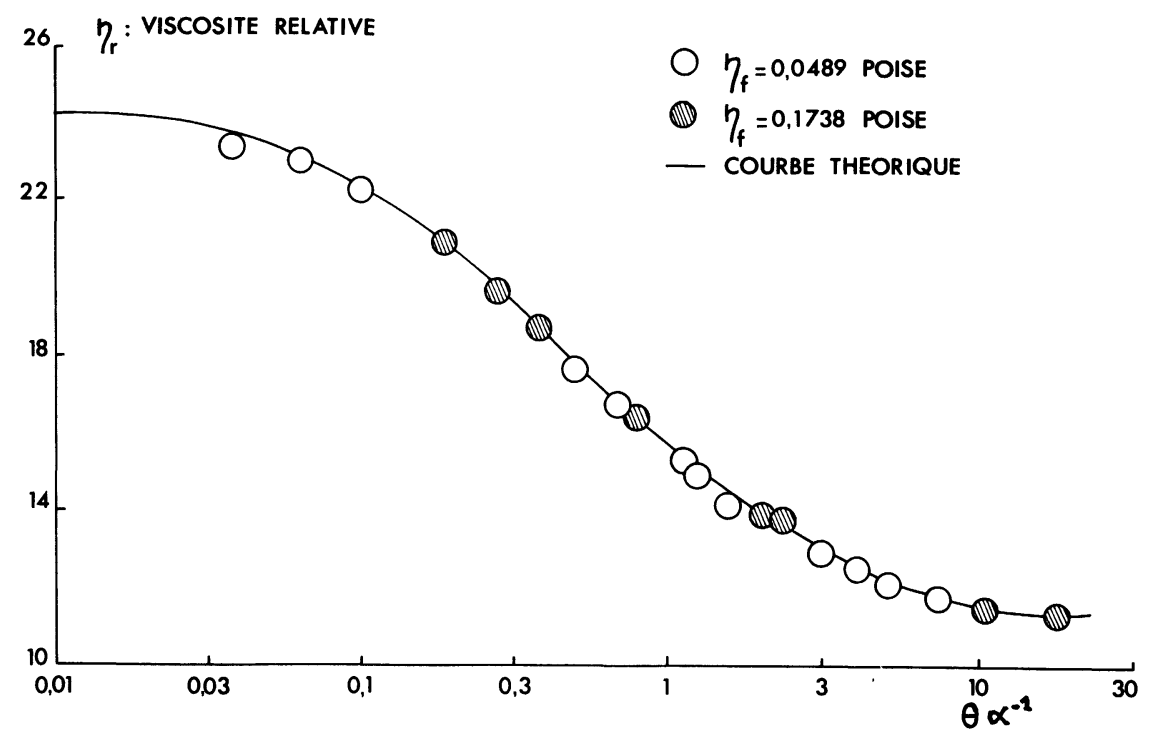

Fig. 1. - Viscosité apparente relative des suspensions de sphères dures en fonction de la contrainte réduite $\theta=\alpha a^{3} \eta_{\mathrm{F}} \dot{\gamma} / k T$. L'expression théorique (10) de la viscosité est alors :

$$
\eta_{\mathrm{r}}=\eta_{1 \mathrm{r}}+\eta_{2 \mathrm{r}}(1+\theta)^{-1} ; \quad \alpha=0,431 .
$$

Les points expérimentaux correspondent à différentes tailles de particules et à deux valeurs très différentes de la viscosité du milieu suspendant. La concentration est $c=0,5$ (d'après les données de Krieger [2]).

[Apparent relative viscosity of hard sphere dispersions versus reduced shear stress $\theta=\alpha a^{3} \eta_{\mathrm{F}} \dot{\gamma} / k T$. The theoretical expression for the bulk viscosity is here (10) :

$$
\eta_{\mathrm{r}}=\eta_{1 \mathrm{r}}+\eta_{2 \mathrm{r}}(1+\theta)^{-1} ; \quad \alpha=0.431
$$

Experimental results correspond to a wide range of particle size and suspending medium viscosity. Particle concentration is $c=0.5$ (from Kriegers' data [2]).]

sphères dures non browniennes doit être voisine de la valeur de la viscosité d'une suspension de sphères dures browniennes obtenue pour de grandes valeurs du taux de cisaillement, l'amplitude du mouvement brownien rotationnel étant alors négligeable devant celle du mouvement induit par les forces hydrodynamiques sur chaque particule.

Les résultats expérimentaux (par exemple ceux d'Eilers [4] sur des sphères de diamètre $D$,

$$
1,6 \mu \mathrm{m}<2 a<8,1 \mu \mathrm{m})
$$

confirment ce point de vue.

Cependant, malgré la dispersion, suivant les auteurs, des résultats expérimentaux concernant les grosses particules, on notera que la viscosité d'une suspension de particules non browniennes est toujours légèrement inférieure à celle des suspensions de particules browniennes à taux de cisaillement infini.

La croissance de la viscosité de suspensions de sphères dures browniennes, lorsque le cisaillement décroît, a été justifiée par Krieger et Dougherty [5] par la formation de doublets de particules initiés par le mouvement brownien et cassés ensuite dans le champ de cisaillement. Krieger a ajusté de très nombreux résultats expérimentaux [2] en faisant varier la viscosité du milieu suspendant et la taille des sphères dures, avec une loi du même type que celle proposée par Williamson [6]

$$
\eta_{\mathrm{r}}=\eta_{\mathrm{r} x}+\frac{\eta_{\mathrm{r} 0}-\eta_{\mathrm{r} x}}{1+\theta}, \quad \theta=\alpha \frac{\tau a^{3}}{k T}
$$

\section{Dans l'expression (1) :}

- $\eta_{\mathrm{r}}$ est la viscosité relative de la suspension; - $\eta_{\mathrm{r} x}$ et $\eta_{\mathrm{r} 0}$ sont respectivement les viscosités relatives à grand taux de cisaillement et à faible taux de cisaillement (dans les expériences de Krieger, la contrainte $\tau=\eta_{1} \dot{\gamma}$ variait dans la gamme : $3 \times 10^{-2}-30$ dyne $\mathrm{cm}^{-2}$;

- $\theta$ est une contrainte relative qui s'exprime en fonction de $\tau=\eta_{\mathrm{F}} \dot{\gamma}\left(\eta_{\mathrm{F}}\right.$ étant la viscosité du milieu suspendant), du rayon $a$ des sphères dures, de la constante de Boltzmann $k$, et de la température absolue $T$, et d'une constante purement numérique $\alpha$. La dépendance en $a^{3}$ rend ce terme très sensible à la dimension des particules, ce qui sépare nettement le comportement (non newtonien) des suspensions de particules browniennes, de celui (newtonien) des suspensions de grosses particules.

La relation semi-empirique (1) ajuste de façon remarquable les résultats expérimentaux. Plus récemment, l'un des auteurs [7] a ajusté les résultats de 
Krieger avec une loi de viscosité déduite d'un principe de dissipation extrémale.

Dans ce qui suit un modèle de fluide micropolaire nous permettra d'établir une expression théorique de la viscosité d'une suspension de sphères dures browniennes. La théorie des fluides micropolaires est une théorie de fluides continus généralisés où un macroélément du fluide, regardé à une échelle plus fine peut se représenter comme une réunion de microéléments : on dit que le milieu possède une microstructure. Les microéléments seront ici les sphères dures. On peut lier au centre d'inertie de chaque sphère un système de trois directeurs; le cas le plus simple envisagé dès 1909 par E. et F. Cosserat [8], celui où la figure formée par les directeurs est invariable, correspond à des microstructures non déformables comme les sphères dures que nous étudions ici. Dans le cas d'un écoulement bidimensionnel entre deux plaques dont l'une est mobile (vitesse $v_{0}$ ) et l'autre fixe, les équations générales des fluides micropolaires se simplifient considérablement (voir par exemple la référence [9]) et les équations décrivant l'écoulement du fluide s'expriment au moyen de deux variables indépendantes, la vitesse $\underline{v}$ et la microrotation $\omega$.

Les équations décrivant l'écoulement stationnaire du fluide sont alors :

- Conservation de la quantité de mouvement :

$\frac{1}{2} \frac{\mathrm{d} v_{x}}{\mathrm{~d} y}=-\Omega_{z}=\frac{1}{2\left(\eta_{1}+\eta_{2}\right)}\left[\tau_{\mathrm{p}}-2 \eta_{2} \omega_{z}\right]$

- Conservation du moment de quantité de mouvement :

$$
\beta \frac{\mathrm{d}^{2} \omega_{z}}{\mathrm{~d} y^{2}}=2 \eta_{2}\left(\omega_{z}-\Omega_{z}\right) .
$$

- Condition de concentration homogène :

$$
\frac{\mathrm{d} c}{\mathrm{~d} y}=0
$$

où $\eta_{1}(c), \eta_{2}(c)$ sont respectivement les viscosités de cisaillement et de rotation, $\beta(c)$ un paramètre homogène au carré d'une longueur, $c$ la concentration, et $\tau_{\mathrm{p}}$ la contrainte à la paroi.

La valeur de la microrotation $\omega(y)$ est donnée par l'équation $(2 b)$ à condition de connaître les valeurs de $\omega$ aux parois. Une condition aux limites classique consiste à annuler $\omega$ à la paroi ; alors l'écart entre la microrotation et la vorticité diffuse à travers l'écoulement sur une longueur supérieure mais du même ordre de grandeur que la microstructure. Dans la situation expérimentale qui nous intéresse, cette longueur est du même ordre de grandeur que le rayon de la sphère dure brownienne, donc négligeable par rapport à la longueur caractéristique de l'écoulement viscosimétrique. Les conditions sur la microrotation aux parois n'ont donc aucune influence sur l'écoulement de la suspension. Si aucun autre facteur n'intervient, la microrotation des sphères est en tout point, autre que les parois, identique à la vorticité du fluide. On peut introduire un couple thermodynamique représentant l'effet de la diffusion rotationnelle, et définir le rapport $\theta^{\prime}$, analogue à un nombre de Peclet, du couple exercé par la particule sur le fluide $\left(\eta_{\mathrm{F}} \dot{\gamma} 4 \pi a^{2}\right) a$ sur le couple thermodynamique $k T / 2$ :

$$
\theta^{\prime}=\frac{8 \pi \eta_{1} \dot{\gamma} a^{3}}{k T}
$$

Suivant Batchelor [10], $\theta^{\prime}$ peut être interprété comme le quotient du temps requis pour qu'une particule prenne une orientation donnée due au mouvement du fluide seul, par le temps requis pour que, sous l'effet du mouvement brownien, une distribution arbitraire d'orientation relaxe vers une distribution uniforme.

Lorsqu'une sphère brownienne test est placée dans un champ de cisaillement pur $\dot{\gamma}$, il est admis généralement que le flux total de particules $J$ à travers la section efficace de collision, introduit dans la théorie de la coagulation de Smoluchowski (11) est la somme du flux $J_{\mathbf{B}}$ dû au mouvement brownien en l'absence de cisaillement et du flux $J_{\mathrm{S}}$ créé par le cisaillement en l'absence du mouvement brownien. Dans ces conditions, le flux $J$ croît linéairement avec le nombre de Peclet de translation $P e_{1}$. Récemment, Van de Ven et Mason [12], ont montré que le taux de collision de sphères dures browniennes, à cisaillement faible, variait comme la racine carrée du nombre de Peclet $P e_{1}$.

On conçoit que lors d'une collision brownienne à laquelle on peut associer une fréquence de collision

$$
f_{\mathrm{B}}=\tau_{\mathrm{B}}^{-1}=\frac{2 c k T}{\pi \eta_{\mathrm{F}} a^{3}}
$$

les particules oublient le gradient de vitesse dans lequel chacune se trouvait individuellement. Sur l'ensemble des collisions browniennes, la microrotation de chacune des particules fluctue alors autour d'une valeur moyenne nulle $\omega=0$. Par contre, lorsque la collision est initiée par le cisaillement avec une fréquence de collision

$$
f_{\Omega}=\tau_{\Omega}^{-1}=\frac{8 c \dot{\gamma}}{\pi}
$$

les particules conservent leur rotation initiale voisine de la vorticité $\Omega$. D'après $(4 a)$ et $(4 b)$, le rapport $f_{\Omega} / f_{\mathrm{A}}$, égal, à un facteur numérique près, au nombre $\theta^{\prime}$, donné en (3), est lié au pourcentage relatif du nombre de particules subissant une collision brownienne par rapport à celles subissant une collision initiée par le cisaillement.

- De l'équation (2a) moyennée, on peut déduire 
la viscosité apparente $\eta$ de la suspension en fonction de la valeur moyenne de la microrotation : $\bar{\omega}$

$$
\eta=\eta_{1}+\eta_{2}\left(1-\frac{\bar{\omega}}{\Omega}\right) .
$$

Cette valeur moyenne $\bar{\omega}$ peut être évaluée de la façon suivante. Considérons les deux états accessibles pour la microrotation :

$$
\omega_{1}=0, \quad \omega_{2}=\Omega .
$$

Le premier état correspond au centre de la distribution des microrotations des particules lors des collisions browniennes et le deuxième état à l'égalité entre la microrotation des particules et la vorticité du fluide. Lorsque la suspension est en écoulement stationnaire ( $\gamma$ fixé), il existe un équilibre dynamique entre les deux populations correspondant à chacun de ces deux états $\omega_{1}=0$ et $\omega_{2}=\Omega$. Soient $n(0)$ et $n(\Omega)$ les fractions numériques correspondantes. La relaxation des particules entre ces deux états est décrite par :

$$
\frac{\mathrm{d} n(0)}{\mathrm{d} t}=\tau_{\mathbf{B}}^{-1}[1-n(0)]
$$

où $\tau_{\mathrm{B}}$ est le temps de relaxation pour que la distribution d'orientation des particules centrées sur $\omega_{2}$ relaxe par diffusion rotationnelle brownienne vers une distribution uniforme correspondant à $\omega_{z}=\Omega$.

D'autre part :

$$
\frac{\mathrm{d} n(\Omega)}{\mathrm{d} t}=\frac{\mathrm{d}[1-n(0)]}{\mathrm{d} t}=\tau_{\Omega}^{-1} n(0)
$$

où $\tau_{\Omega}$ est le temps requis pour qu'une particule prenne une orientation donnée due au mouvement du fluide seul.

Dans un écoulement stationnaire

$$
\tau_{\mathrm{B}}^{-1}[1-n(0)]-\tau_{\Omega}^{-1} \cdot n(0)=0 .
$$

On en déduit les populations $n(0)$ et $n(\Omega)$ :

$$
n(0)=\frac{\theta}{1+\theta}, \quad n(\Omega)=\frac{1}{1+\theta}
$$

avec

$$
\theta=\frac{\tau_{\mathrm{B}}}{\tau_{\Omega}}=\frac{\alpha \tau a^{3}}{k T}
$$

et la valeur moyenne de la microrotation :

$$
\bar{\omega}=\sum_{\substack{i \\ i=1,2}} \omega_{i} n\left(\omega_{i}\right)=\Omega n(\Omega)
$$

d'où

$$
\bar{\Omega}=n(\Omega)=\frac{1}{1+\theta} .
$$

On déduit alors, en reportant (9) dans (5), une expression théorique de la viscosité apparente :

$$
\eta=\eta_{1}+\frac{\eta_{2}}{1+\theta}, \quad \theta=\frac{\alpha \tau a^{3}}{k T} .
$$

C'est très précisément la relation empirique de Williamson et de Krieger où les valeurs de la viscosité à faible cisaillement et à taux de cisaillement infini ont maintenant une définition précise : ce sont respectivement la somme $\eta_{1}+\eta_{2}$ de la viscosité de cisaillement et de la viscosité de rotation et la viscosité de cisaillement $\eta_{1}$.

On a ainsi un moyen d'accéder expérimentalement à la viscosité de rotation $\eta_{2}$. L'étude des résultats expérimentaux tirés de $[2,5]$ permet de tracer les courbes représentant les viscosités de cisaillement et de rotation en fonction de la concentration volumique des particules (Fig. 2).

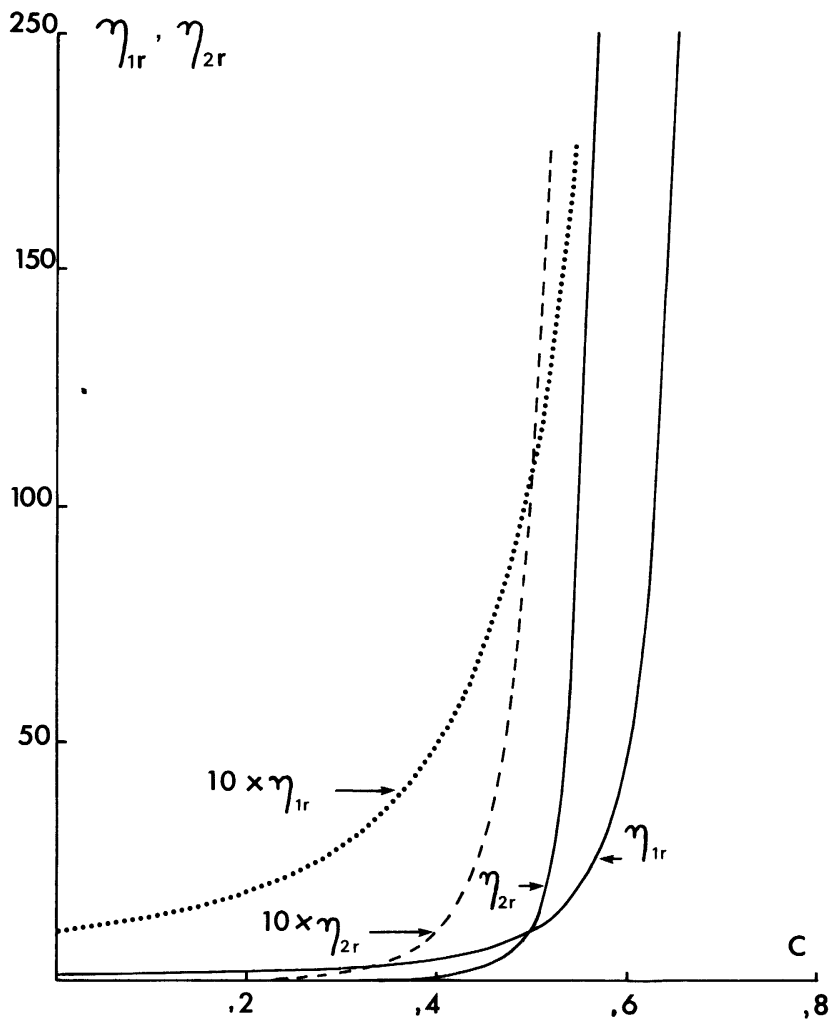

Fig. 2. - Viscosités relatives de cisaillement $\eta_{1 \mathrm{r}}$ et de rotation $\eta_{2 \mathrm{r}}$ déduites en ajustant les résultats expérimentaux de Krieger [2] grâce à l'expression théorique (10), en fonction de la concentration de particules. La viscosité de rotation est négligeable pour des concentrations inférieures à 0,25 .

[Relative shear viscosity $\eta_{1 \mathrm{r}}$ and rotation viscosity $\eta_{2 \mathrm{r}}$ versus particle concentration obtained by interpreting Krieger's experimental results in terms of our theoretical expression (10). As the particle concentration is decreased below 0.25 , rotation viscosity becomes very low.]

Les résultats expérimentaux ajustés par une méthode des moindres carrés conduisent aux expressions suivantes de la viscosité apparente relative $\eta_{\mathrm{r}}$, de la 
viscosité de cisaillement relative $\eta_{\mathrm{r} 1}$ et de la viscosité de rotation $\eta_{\mathrm{r} 2}$ en fonction de la concentration $c$ :

viscosité apparente

$$
\eta_{\mathrm{r}}=\eta_{\mathrm{r} 1}+\frac{\eta_{\mathrm{r} 2}}{1+0,431 \frac{\tau a^{3}}{k T}}
$$

viscosité de cisaillement

$$
\eta_{\mathrm{r} 1}=\left(1-\frac{c}{0,68}\right)^{-1,82}
$$

viscosité de rotation

$$
\eta_{\mathrm{r} 2}=\eta_{\mathrm{r} 0}-\eta_{\mathrm{r} 1}
$$

où

$$
\eta_{\mathrm{r} 0}=\left(1-\frac{c}{0,57}\right)^{-1,50} .
$$

Pour conclure, l'expression théorique de la viscosité apparente relative est une équation d'état rhéologique du fluide s'exprimant au moyen de trois grandeurs adimensionnées. Pour une concentration volumique et une température données, on obtient une seule courbe $\eta(\theta)$ quelle que soit la taille des particules et la viscosité du milieu suspendant.

Cela est parfaitement confirmé par l'ajustement par une seule courbe de nombreux résultats expérimentaux de Krieger [2]. D'autre part, l'expression théorique de la viscosité apparente donne accès à une grandeur importante dans la théorie des milieux continus généralisés, la viscosité de rotation, dont la variation en fonction de la concentration était jusqu'à présent inconnue.

\section{Bibliographie}

[1] Woods, M. E., Dodge, J. S., Pierce, P. E., PaPir, Y. S. and Krieger, I. M., J. Paint Technol. 40 (1968) $545 ; 42$ (1970) $71,572$.

[2] Krieger, I. M., Adv. Colloild Interface Sci. (1972) 111-136.

[3] Goldsmith, H., Mason, S. in Rheology : theory and application Eirich. ed. (Acad. Press) 1964, vol. 4, pp. 191193.

[4] Eilers, H., Kolloïd-Z. 97 (1941) 913 ; 102 (1943) 154.

[5] Krieger, I. M. and DougherTy, Y. T. J., Trans. Soc. Rheol. 3 (1959) $137-152$.

[6] Williamso $\backslash$. R. V., J. R/ı'ol. 1 (1930) 283.
[7] Quemada, D., Rheol. Acta 17 (1978) 643-653.

[8] CosserAT, E. et F., Théorie des corps déformables (Hermann et fils, Paris) 1909.

[9] ERINGEN, C. J., of Math. and Mech. Vol. 16 (1966) № 1, pp. 1-18.

[10] Batchelor, G. K., Theoretical and Applied Mechanics (Wt Koiter ed.) 1976, pp. 33-55.

[11] Smoluchowsky, M. Von, Z. Phys. Chem. 91 (1917) 129.

[12] Van De Ven, T. G. and Mason, S. G., Colloid and Polym. Sci. 255 (1977) 794-804. 